\title{
ПОСТОЯНСТВО ТИПА ПО ВАНХЕККЕ И НАТУРАЛЬНЫЕ ПРОСТРАНСТВЕННЫЕ ФОРМЫ
}

\author{
(Представил А. Хумал)
}

Комплексные пространственные формы, или келеровы многообразия постоянной голоморфной секционной кривизны (короче, $H S$-кривизны), являются одним из наиболее простых и в то же время очень интересных объектов геометрии келеровых многообразий. Хорошо известна их полная классификация [ $\left.{ }^{1}\right]$. Недавно рядом авторов была получена полная классификация приближенно келеровых многообразий постоянной $H S$-кривизны $\left[{ }^{2,3}\right]$. Все эти многообразия имеют постоянный в смысле Грея тип [ $\left.{ }^{4}\right]$. Л. Ванхекке обобшил понятие постоянства типа на произвольные почти эрмитовы многообразия. Это дало ему основание определить обобщенные комплексные пространственные формы $M(\mu, \alpha)$ как почти эрмитовы многообразия постоянного типа $\alpha$ постоянной $H S$-кривизны $\mu\left[{ }^{5}\right]$. Л. Ванхекке изучил ряд свойств таких многообразий, в частности, структуру их тензора кривизны в случае его $J$-инвариантности $\left[{ }^{6}\right]$. Изучением таких многообразий занимались также С. Саваки и К. Секнгава, которые, в частности, доказали, что обобщенная комплексная пространственная форма нулевого постоянного типа является келеровым многообразием [ $\left.{ }^{7}\right]$.

В настоящей работе вводится в рассмотрение класс дифференциально-геометрических структур, существенно обобщающих понятие почти эрмитовой структуры, и строится аппарат их исследования, позволяющий преодолеть многие традиционные трудности эрмитовой геометрии. C его помощью получена полная классификация натуральных пространственных форм, естественно обобщающих понятие обобщенных комплексных пространственных форм, в случае расшепляемых редуктивных обобщенных квазикелеровых многообразий, что значительно усиливает большинство имеющихся в этом направлении результатов.

\section{Q-алгебры}

Пусть $\mathfrak{\Omega}-$ кольцо с инволюцией, $V-$ (левый $) \Re$-модуль, \langle\rangle$-$ невырожденная эрмитова форма на $V$, которая называется также скалярным произведением или метрикой. Назовем 〈,〉 расщепляющим скалярным произведением, если для всякого подмодуля $U \subset V$, ограничение метрики на который невырождено, $V=U \oplus U^{\perp}$, где $U^{\perp}-$ ортогональное дополнение $U$. Назовем расщепляющее скалярное произведение строго невырожденным, если для всякого подмодуля $U \subset V$ ограничение метрики на $U$ невырождено.

Опредделение 1. Эрмитову $Q$-алгебру $V$ определим следующцими свойствами:

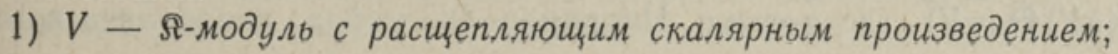


2) $V-\Omega$-алгебра с бинарной операцией * : $V \times V \rightarrow V$, антилинейной по каждому аргументу;

3) $\langle X * Y, Z\rangle+\langle\overline{Y, X * Z\rangle}=0(X, Y, Z \in V)$.

Определим в $V$ операции альтернированного $\left({ }^{\circ}\right)$ и сопряженного $(\nabla)$ умножений соотношениями $X \circ Y=X * Y-Y * X$ и $\langle X * Y, Z\rangle=$ $=\langle\overline{X, Y \nabla Z}\rangle(X, Y, Z \in V)$ соответственно. Очевидно, эти операции антикоммутативны. $Q$-алгебру $V$ назовем абелевой, если $V * V=\{0\}$, $A$-алгеброй, если $X \circ Y=Y \nabla X(X, Y \in V)$, полупростой, если она не содержит ненулевых двусторонних абелевых идеалов, и правильной, если каждый ее левый идеал является двусторонним идеалом. Будем говорить, что $Q$-алгебра $V$ распадается в прямое произведение идеалов

$J_{1}, \ldots, J_{r}$, если $V=J_{1} \otimes \ldots \oplus J_{r}$. Введем обозначение: $V=J_{1} \dot{\times} \ldots \dot{\times} J_{r}$. Очевидно, при этом $J_{i} * J_{j}=\{0\} \quad(i \neq j)$. Правильную $Q$-алгебру назовем редуктивной, если она распадается в прямое произведение абелева идеала и полупростого идеала. Например, нетрудно показать, что правильная $Q$-алгебра со строго невырожденной метрикой является редуктивной.

Те орем а 1. Пусть $V-$ редуктивная $Q$-алгебра; $V=A \dot{\times} B$, где $A-$ абелев, $B$ - полупростой идеаль. Тогда левый аннулятор $V$ совпадает c аннулятором $\mathfrak{A}(V)$ Q-алгебры $V$ и совпадает с $A$, a $B-$ производный идеал Q-алгебры $V$, т. е. $B=V * V$. При этом $A \perp B$.

Доказательство схоже с доказательством аналогичного утверждения для редуктивных алгебр Ли [ $\left.{ }^{8}\right]$.

Пусть $V^{R}-Q$-алгебра с тривнальной инволюцией - тождественным отображением - над коммутативным ассоциативным кольцом ్ с единицей, $J_{0}=\mathrm{id}, J_{1}, \ldots, J_{r}-$ линейно независимые попарно коммутирующие эндоморфизмы модуля $V^{R}$, являющиеся вместе со своими квадратами образующими подалгебры $\AA$ алгебры всех эндоморфизмов модуля $V^{R}$ и удовлетворяющие условиям:

1) $J_{k}(X) * Y=X * J_{k}(Y)=-J_{k}(X * Y)$; 2) $\left\langle J_{k} X, Y\right\rangle+\left\langle X, J_{k} Y\right\rangle=0$;

3) $\bigcap_{k=1}^{r} \operatorname{ker} J_{k} \subset \mathfrak{U}\left(V^{R}\right) \quad\left(k=1, \ldots, r ; X, Y \in V^{R}\right)$.

Введем в алгебре $\mathfrak{\Omega}$ инволюцию соотношениями $\bar{J}_{0}=J_{0}, \bar{J}_{k}=-J_{k}(k=1, \ldots, r)$. Тогда $V^{R}$ можно рассматривать как $\Re$-модуль. Введем в нем форму $H(X, Y)=\sum_{k=0}^{r}\left\langle X, J_{k}^{3} Y\right\rangle J_{k}$. Тройка $\left\{V^{R}, H, *\right\}$ определяет $Q$-алгебру $V$ над $\Omega$, которую мы назовем натуральной $Q$-алгеброй ранга $r$, причем $Q$-алгебру $V^{R}$ назовем ее $\Im$-формой, а эндоморфизмы $J_{1}, \ldots, J_{r}-$ структурными аффинорами. Например, Q-алгебры над полем C комплексных чисел, над кольцом D двойных чисел и над полем $\mathbf{R}$ вещественных чисел - натуральные $Q$-алгебры ранга $1,-1$ и 0 соответственно, которые мы назовем $Q$-алгебрами эллиптического, гиперболического и параболического типов соответственно.

Пусть $V-$ натуральная $Q$-алгебра над $\mathfrak{R}$. Легко видеть, что в модуле $V^{\Re}=V^{R} \otimes \Omega$ естественно определены оператор сопряжения $\tau$ и структура натуральной $Q$-алгебры над $\Re$. $Q$-алгебру $V$ назовем расщепляемой, если существует вложение $Q$-алгебр $\sigma: V \rightarrow V \Omega$ такое, что $V^{\Re}=(\sigma V) \oplus(\bar{\sigma} V)$, где $\bar{\sigma}=\tau \cdot \sigma$. Например, $Q$-алгебра параболического 
типа, очевидно, нерасщепляема, а $Q$-алгебры эллиптического или гиперболического типов расшепляемы, причем $\sigma=\left(\mathrm{id}-1-j^{3} J \Re\right) / 2$, где $j^{2}=\mp 1$ соответственно, $J^{\Re}=J \otimes \mathrm{id}_{\Re}$.

Теорем а 2. Пусть $V$ - расшепляемая $Q$-алгебра над $\Omega, L-\Omega$-модуль. Существует естественная биекция между множеством L-значных $r$-линейных отображений $V^{\Omega}$ и множеством упорядоченных наборов $2^{r}$ L-значных форм на $V$, линейных либо антилинейных по различным наборам аргументов.

Доказательство следует из определения расщепляемой $Q$-алгебры. Назовем набор этих форм спектром указанного отображения.

\section{2. Обобщенные почти эрмитовы структуры}

Пусть $M$ - гладкое многообразие, $\mathscr{X}(M)$ - алгебра Ли гладких векторных полей на $M$. Все многообразия, тензорные поля и т. п. объекты предполагаются гладкими класса $C^{\infty}$.

О пр еделени е 2. Обобщенной почти эрмитовой структурой (короче, $G A H$-структурой) ранга $r$ на $M$ называется совокупность $\left\{\langle,\rangle ; J_{1}, \ldots\right.$, $\left.\ldots, J_{r}, T\right\}$, где $\langle\rangle=$,$g - псевдориманова метрика на M, J_{1}, \ldots, J_{r}-$ линейно независимье в каждой точке многообразия тензорь типа $(1,1)$ на $M$, называемые структурными аффинорами, являющиеся вместе со своими квадратами и тождественньм аффинором образующими подалгебры алгебры всех эндоморфизмов касательного пучка многообразия, T-тензор типа $(2,1)$ на $М$, называемьй композиционнььм. При этом:

1) $\left\langle J_{i} X, Y\right\rangle+\left\langle X, J_{i} Y\right\rangle=0$; 2) $T\left(J_{i} X, Y\right)=T\left(X, J_{i} Y\right)=-J_{i} T(X, Y)$;

3) $T_{X} g=0$; 4) $\bigcap_{i=1}^{r} \operatorname{ker} J_{i} \subset \operatorname{ker} T$; 5) $J_{i} J_{j}=J_{j} J_{i}$

$(i, j=1, \ldots, r ; X, Y \in \mathfrak{X}(M))$.

Здесь $T_{X} Y=T(X, Y)$, оператор $T_{X}$ отождествляется с порожденным им дифференцированием тензорной алгебры многообразия. Многообразие, наделенное $G A H$-структурой, назовем $G A H$-многообразием. $G A H$-структура называется обобшенной квазикелеровой, или $G Q K$ структурой, если $\nabla_{X} J_{i}+T_{X} J_{i}=0(i=1, \ldots, r ; X \in \mathfrak{X}(M))$, где $\nabla$ риманова связность на $M$.

Введем в модуле $\mathfrak{X}(M)$ операцню «*» по формуле $X * Y=T(X, Y)$, $(X, Y \in \mathfrak{X}(M))$. С учетом (1) убеждаемся, что эта операция определяет в модуле $\mathfrak{X}(M)$ структуру эрмитовой натуральной $Q$-алгебры $\mathfrak{B}$ ранга $r$ с метрикой $H(X, Y)=\sum_{k=0}^{r}\left\langle X, J_{k}^{3} Y\right\rangle J_{k}$, где $J_{0}=\mathrm{id}$, причем в касательном пространстве $T_{p}(M)$ в каждой точке $p \in M$ также индуцируется структура эрмитовой натуральной $Q$-алгебры ранга $r$, которую мы обозначим через $\mathfrak{B}_{p}$. Назовем $Q$-алгебру $\mathfrak{B}$ (соответственно, $\mathfrak{B}_{p}$ ) присоединенной $\mathrm{K}$ многообразию $M$ (в точке $p$ ). Условимся фиксировать в названии $G A H$ многообразия соответствующее свойство присоединенной $Q$-алгебры. Например, редуктивным $G A H$-многообразием назовем $G A H$-многообразие, присоединенная $Q$-алгебра которого редуктивна. $G Q K$-многообразие, присоединенная $Q$-алгебра которого антикоммутативна (абелева, $A$-алгебра), назовем, соответственно, обобщенным приближенно келе- 
ровым (GNK-), обобщенным келеровым (GK-) и обобщенным почти келеровым (GAK-) многообразием.

Примером $G A H$-структуры служит структура, индуцированная на метрических $f$-многообразиях [ $\left.{ }^{9}\right]$, наиболее важными примерами которых являются почти эрмитовы и почти контактные многообразия. Напомним, что метрической $f$-структурой на гладком многообразии $M$ называется пара $\{f,\langle\rangle$,$\} , где f-$ тензор типа $(1,1) ; f^{3}+\varepsilon f=0 ;\langle\rangle-$. псевдориманова метрика такая, что $\left\langle f^{2} X, f^{2} Y\right\rangle=\varepsilon\langle f X, f Y\rangle(X, Y \in \mathfrak{X}(M))$, где $\varepsilon= \pm 1$ в эллиптическом (соответственно, гиперболическом) случае. Значение почти эрмитовых структур в классе метрических $f$-структур определяется тем, что их присоединенная $Q$-алгебра расщепляема. Тензор $T$, необходимый для определения $G A H$-структуры на метрическом $f$-многообразии, определяется формулой

$$
T(X, Y)=\left\{N(X, Y)-N^{*}(X, Y)-N^{*}(Y, X)\right\} / 2,
$$

где $N(X, Y)=-f^{2}[f X, f Y]+f^{2}\left[f^{2} X, f^{2} Y\right]+f\left[f X, f^{2} Y\right]+f\left[f^{2} X, f Y\right]$;

$\langle N(X, Y), Z\rangle=\left\langle X, N^{*}(Y, Z)\right\rangle(X, Y, Z \in X(M)$, Г, $1-$ скобка Ли). В случае почти эрмитова многообразия тензор $N$ совпадает с классическим тензором Нейенхейса.

Пусть $M$ - расщепляемое $G Q K$-многообразие. В этом случае в каждой точке $p \in M$ отображение $\sigma$ (см. раздел 1) индуцирует вложение множества унитарных реперов модуля $\mathfrak{B}_{p}$ во множество всех реперов модуля $\mathfrak{B}_{p}^{\Omega}$. В объединении $\Re$ по всем точкам $p \in M$ реперов модулей $\mathfrak{g}_{p}^{\Omega}$ стандартным образом вводится структура главного расслоенного пространства со стандартной проекцией, а в образе указанного вложения - его подрасслоения, т. е. G-структуры (5. Записывая структурные уравнения Картана этой $G$-стрvктуры, нетрудно установнть. что спектр тензора $R$ кривизны римановой связности зависит от композишионного тензора и его ковариантных производных, а также от 3-формы $A$. co значениями в $\mathfrak{B}$ на модуле $\mathfrak{B}$, линейной и симметричной по первым двум аргументам и антилинейной по третьему, которую мы назовем тензором кривизны присоединенной $Q$-алгебры.

\section{3. Постоянство типа по Ванхекке}

О пределение 3 . Пусть $J-$ структирный аффинор $G A H$-многообразия М. Введем в рассмотрение биквадратичнию форми $\lambda(X, Y)=$ $=\left\langle J^{2} R(X, Y) X, J^{2} Y\right\rangle-\langle J R(J X, J Y) X, J Y\rangle(X, Y \in \mathfrak{X}(M))$. Будем говорить, что $M$ имеет постоянный по Ванхекке тип а относительно аффинора $J$,

$$
\begin{aligned}
& \text { если } \lambda(X, Y)=\alpha\|J X\|^{2}\|J Y\|^{2}\left(X, Y \in \mathfrak{X}(M) ; \quad\langle X, Y\rangle=\left\langle X, J^{3} Y\right\rangle=0 ; \quad \alpha \in\right. \\
& \left.\in C^{\infty}(M)\right) .
\end{aligned}
$$

Учитывая строение спектра тензора кривизны, нетрудно убедиться, что расщепляемое $G Q K$-многообразие $M$ имеет постоянный тип тогда и только тогда, когда $\nabla_{X} T+T_{X} T=0$ и $H(X \circ Y, X \nabla Y)=$ $=\alpha H(X, X) H(Y, Y) \quad(X, Y \in \mathfrak{B} ; H(X, Y)=0)$. Поляризацией этого соотношения с учетом невырожденности метрики несложно проверить, что оно равносильно соотношению

$$
(X \circ Y) * Z=\alpha\{H(X, Z) Y-H(Y, Z) X\} \quad(X, Y, Z \in \mathfrak{B}) .
$$

В частности, постоянство типа расщепляемого $G Q K$-многообразия не зависит от выбора структурного аффинора. Далее, из (2) следует, что нулевое постоянство типа расщепляемого $G A K$-многообразия со 
знакоопределенной метрикой равносильно соотношению $\|X \circ Y\|^{2}=0$ и абелевости присоединенной $Q$-алгебры. Получен результат:

T ео рем а 3. Расщепляемое GAK-многообразие со знакоопределенной метрикой нулевого постоянного типа является GK-многообразием.

Эта теорема обобщает известный результат Гольдберга $\left[{ }^{10}\right]$. Заметим, что утверждение теоремы не переносится на расщепляемые $G Q K$-многообразия, так как многообразие $S^{2} \times \mathrm{R}^{4}$, снабженное канонической квазикелеровой структурой, индуцированной 3-векторными произведениями алгебры Кели, некелерово, хотя и имеет. нулевой постоянный тип, поскольку является $F$-пространством $\left[{ }^{11}\right]$.

Определение 4. Пусть $J$ - структурный аффинор GAH-многообразия М. Будем говорить, что $X \in \mathfrak{X}(M)$ удовлетворяет условию $J$-невырожденности, $и$ обозначим $X \in \mathfrak{X}_{J}(M)$, если бивектор $X \wedge J X$ неизотропен в каждой точке многообразия М. Это, очевидно, равносильно тому, что векторы $X и$ ЈХ неколлинеарны и неизотропны в каждой точке многообразия. Голоморфной секционной кривизной (короче, HS-кривизной) $H(X) G A H$-многообразия $M$ относительно структурного аффинора $J$ в направлении $X \in \mathfrak{X}_{J}(M)$ назьвается кривизна в двумерном направлении $X \wedge J X, T$. $e$.

$$
\langle R(X, J X) X, J X\rangle=H(X)\|X\|^{2}\|J X\|^{2} .
$$

Стандартным образом определяется точечное и глобальное постоянство $H S$-кривизны на $M\left[{ }^{12}\right]$ как постоянство относительно каждого структурного аффинора с одной и той же кривизной.

Tеорема 4. Расшепляемое $G Q K$-многообразие имеет точечно постоянную $H S$-кривизну $4 \mu$ тогда и только тогда, когда

$$
A(X, Y, Z)=\{Z \nabla(X * Y+Y * X)-\mu H(X, Z) Y-\mu H(Y, Z) X\} / 2 .
$$

При этом справедливо тождество

$$
Z \nabla(X * Y+Y * X)+Z *(X * Y+Y * X)=0 \quad(X, Y, Z \in \mathfrak{B}) .
$$

Доказательство основано на поляризации (3) с учетом строения спектра тензора $R$.

\section{4. Натуральные пространственные формы}

О п реде лен ие 5 . GAH-многообразие постоянного типа а постоянной HS-кривизны $4 \mu$ относительно структурного аффинора J назовем натуральной пространственной формой.

Из сказанного следует, что расщепляемое $G Q K$-многообразие будет натуральной пространственной формой тогда и только тогда, когда

$$
\begin{aligned}
2 A(X, Y, Z)= & -Z *(X * Y+Y * X)-\mu\{H(X, Z) Y+H(Y, Z) X\}, \\
& (X \circ Y) * Z=\alpha\{H(X, Z) Y-H(Y, Z) X\} .
\end{aligned}
$$

При этом $Z \nabla(X * Y+Y * X)+Z *(X * Y+Y * X)=0 \quad(X, Y, Z \in \Re)$.

С учетом результатов $\left[{ }^{13}\right]$ получаем:

Т еор ем а 5. Расщепляемая квазикелерова натуральная пространственная форма со знакоопределенной метрикой является локально однородным редуктивным пространством.

Скалярно умножая обе части последнего соотношения на $\mathbb{W} \in \mathfrak{B}$, после простых преобразований и переобозначений, а также с учетом невырожденности метрики получим, что $Z \nabla(X \circ Y)-Z *(X \circ Y)=0$, 
откуда, в частности, следует, что $(X \circ Y) *(X \circ Y)=0 \quad(X, Y, Z \in \Re)$. С учетом этого, полагая в (4б) $Z=X \circ Y$, получим, что при $\alpha \neq 0$ $H(X, X \circ Y) Y=H(Y, X \circ Y) X$. Если $X$ и $Y$ линейно независимы, тогда очезидно $X * X=0$, т. е. $M-G N K$-многообразие. Используя (4a) и структуру спектра тензора $R$ расщепляемого $G Q K$-многообразия, можно показать, что $M$ - многообразне постоянной кривизны, положительной в случае знакоопределенной метрики, a его присоединенная $Q$-алгебра трехмерна в каждой точке. В случае $\alpha=0$ соотношение (4б) примет вид $(X \circ Y) * Z=0$. Но тогда $W *(X \circ Y)=W \nabla(X \circ Y)=$ $=-(X \circ Y) \nabla W=(X \circ Y) * W=0$, где $W=Z * T+T * Z(X, Y, Z, T \in$ $\in \mathfrak{B})$. С другой стороны, $(Z \circ T) *(X \circ Y)=0$, откуда $(Z * T) *$

$*(X \circ Y)=0$. Но ввиду редуктивности $\mathfrak{B}=\mathfrak{U}(\mathfrak{B}) \dot{X}(\mathfrak{B} * \mathfrak{B})$ и, следовательно, $\mathfrak{B} \circ \mathfrak{B} \subset \mathfrak{A}(\mathfrak{B})$. В то же время $\mathfrak{B} \circ \mathfrak{B} \subset \mathfrak{B} * \mathfrak{B}$, ввиду чего $\mathfrak{B} \circ \mathfrak{B} \subset \mathfrak{U}(\mathfrak{B}) \cap(\mathfrak{B} * \mathfrak{B})=\{0\}$, т. е. $\mathfrak{B}-$ коммутативная и, значит, абелева $Q$-алгебра, а $M-G K$-многообразие постоянной $H S$-кривизны. Понимая под эквивалентностью $G A H$-многообразий их диффеоморфизм, индуцирующий изоморфизм $G A H$-структур этих многообразий, получаем следующую классификацию расщепляемых редуктивных квазикелеровых натуральных пространственных форм:

Т еорема 6. Расщепляемая редуктивная квазикелерова натуральная пространственная форма локально эквивалентна одному из следующци GAH-многообразий: 1. Комплексному псевдоевклидову пространству $\mathrm{C}_{k} ;$ 2. Комплексному псевдопроективному пространству С $\mathrm{P}_{k}$; 3. Комплексному псевдогиперболическому пространству $\mathrm{CD}_{n}^{n_{n}} ;$ 4. Двумерному келерову многообразию $\mathrm{M}^{2}$; 5. Двойному евклидову пространству $\mathbf{R}^{n} \overline{\bar{X}} \mathbf{R}^{n} ;$ 6. Многообразию нуль-пар вещественного проективного пространства; 7. Двойному одномерному многообразию $M^{1} \bar{X} M^{1}$; 8. Произведению многообразий типов 1 и 5; 9. Шестимерному многообразию постоянной кривизны, положительной в случае знакоопределенной метрики.

Здесь под двойным многообразием $M \bar{X} M$ понимается многообразие $M \times M$, снабженное канонической паракелеровой структурой $\left[{ }^{14}\right]$; $k$ означает индекс метрики многообразия.

Доказательство в случаях $1-8(\alpha=0)$ сводится к анализу структурных уравнений Картана $G$-структуры $(\mathfrak{G}$, совпадающих с уравнениями Маурера-Картана фундаментальной группы изометрий соответствуюшего однородного пространства, и рассмотрено выше в случае $9(\alpha \neq 0)$.

\section{Л И ТЕРА Т У Р А}

1. L i berm a nn, P., C. r. Acad. sci., 239, № 23, 1579-1581 (1954).

2. Gr a y, A., C. r. Acad. sci., 279, № 22, $797-800$ (1974).

3. К и риченко В. Ф., Мат. заметки, 19 , № $5,797-800$ (1974).

4. Gr a y, A.. Tohoku Math. J., 21, № 4, 614-620 (1969).

5. V a n hecke, L., Hokkaido Math. J., 6, № 1, 31-38 (1977).

6. V a nhecke, L., Rend. mat., 10, № 1, 75-86 (1977).

7. S a waki, S... Sekig a w a, K., J. Different. Geom., 9, № 1, 123-134 (1974).

8. Б у р баки Н., Группы Ли и алгебры Ли, I-III, М., «Мир», 1976.

9. B I a i r, D. E., J. Different. Geom., 4, № 2, 155-167 (1970).

10. Gold berg. S. I., Proc. Amer. Math. Soc., 21, 96-100 (1969).

11. Gr a y, A., Ill. J., Math., 10,353-366 (1966).

12. G r a y, A., J. Different. Geom.. 4, № 3, 283-309 (1970).

13. К и р и чен о В. Ф., Докл АН СССР, 252, № 2, 191-193 (1980).

14. Ros c a, R., C. r. Acad. sci., A B 287, № 7, A539-A541 (1978). 


\section{KIRITSENKO}

\section{VANHECKE TUOPI PUSIVUSED JA LOOMULIKUD RUUMIVORMID}

Artiklis on vaadeldud diferentsiaalgeomeetrilisi struktuure, mis oluliselt üldistavad peaaegu Hermite'i struktuuri mõistet. On välja töötatud nimetatud struktuuride uurimise meetod ja koostatud loomulike ruumivormide täielik klassifikatsioon. Need ruumivormid üldistavad Vanhecke poolt kasutuselevõetud üldistatud komplekssete ruumivormide mõistet reduktiivsete lahutuvate üldistatud kvaasi-Kaehleri muutkondade korral.

\section{KIRITSENKO}

\section{VANHECKE'S CONSTANCY OF TYPE AND NATURAL SPACE FORMS}

Complex space forms, or Kaehler manifolds with constant holomorphic sectional curvature are one of the simplest and, on the other hand, one of the interesting objects of Kaehlerian geometry. Their complete classification is well known. Recently some authors received a complete classification of nearly Kaehler manifoids with constant holomorphic sectional curvature. All these manifolds have constant type in the sense of A. Gray. L. Vanhecke generalized the notion of constancy of type for arbitrary almost Hermitian manifolds, and defined generalized complex space forms $M(\mu, \alpha)$ as almost Hermitian manifolds with constant type $\alpha$ and with constant holomorphic sectional curvature $\mu$. Such manifolds were considered by L. Vanhecke, S. Sawaki, K. Sekigawa and some other authors.

In the present paper, the new class of differential-geometrical structures on smooth manifolds is introduced, which essentially generalizes a notion of almost Hermitian structure, and a method of investigation of such structures is constructed. Due to this method a complete classification of natural space forms is received, which naturally generalizes a notion of generalized complex space forms in the case of splitting reductive generalized quasi-Kaehler manifolds. This generalizes and intensifies most of the known results on this topic. 